Second Meeting, December 9th, 1887.

W. J. Macdonald, Esq., M.A., F.R.S.E., President, in the Chair.

\title{
A Method of Transformation in Geometry.
}

By R. E. Allakdice, M.A.

In spherical, as in plane geometry, transformation by inversion and by similar figures may be used for the deduction of new theorems from known ones, the two methods, however, in the former case becoming identical; and like all other propositions and methods in spherical geometry, these methods of transformation may be dualized. The transformation indicated as the dual of these in the case of the spherical surface is also applicable in plano. The sequel is an account of this method and of some results that may be obtained by means of it.

Let XY (fig. 5) be a fixed straight line, $P$ the curve to be transformed, $A B$ a tangent to $P$ meeting $X Y$ in $B$ and making with $\mathrm{XY}$ an angle $\theta, \mathrm{BC}$ a straight line making with $\mathrm{XY}$ an angle $\phi$; then if $\theta$ and $\phi$ are connected by the relation $\tan \frac{1}{2} \phi=k \tan \frac{1}{2} \theta$, the envelope of $\mathrm{BC}$ is the transformed curve. This transformed curve may be called, for reference, the second inverse of $P$. [The phrase "second inverse" of $P$ would naturally mean the inverse of the inverse of $P$; but, as this is the curve $P$ itself, the phrase is not much used in this sense, and may therefore be employed to designate the curve obtained from $\mathbf{P}$ by the method of tranformation that is dual to the method of inversion.]

The second inverse of a circle is another circle such that the axis of transformation is the radical axis of the two circles.

This theorem is most easily established by proving first the converse, which is as follows:- 
Let $\mathbf{P}$ (fig. 6) be a point in the radical axis of two circles; PQ and $P R$ tangents to these circles; to prove that the ratio $\tan \frac{1}{2} Q P E$ : $\tan \frac{1}{2} R P E$ is constant, independent, that is, of the position of $P$.

Let $P C$ and $P D$, the bisectors of the angles at $P$, meet the central axis of the circles in $C$ and $D$ respectively; then the theorem is equivalent to this, that the ratio CB : ED is constant.

Let QA and PC meet in $O$.

Since $\angle \mathrm{CPQ}=\angle \mathrm{CPE}, \therefore \mathrm{AC}=\mathrm{AO}$.

Now

$\mathbf{C E}: \mathbf{E P}=\mathbf{Q O}: \mathbf{Q P}$, $\therefore \quad \mathrm{CE}: \mathrm{CE}+\mathrm{QA}+\mathrm{AC}=\mathrm{EP}: \mathrm{EP}+\mathrm{QP}$,

$\therefore \quad$ CE : EH $=E P: E P+Q P$;

similarly $\mathrm{DE}: \mathrm{EK}=\mathrm{EP}: \mathrm{EP}+\mathrm{RP}$

$=E P: E P+Q P$.

$\therefore \quad$ CE $: \mathrm{DE}=\mathrm{EH}: \mathbf{E K}$

$=$ constant.

The converse of this theorem is obviously true; that is, if the circle $A$ and the line $P E$ be given and if $P R$ be always drawn so that $\tan \frac{1}{2} \mathrm{RPE}=k \tan \frac{1}{2} \mathrm{QPE}$, where $k$ is a constant, then $\mathrm{PR}$ will envelope a circle, PE will be the radical axis of this circle and the given circle, and the ratio $\mathrm{EK}: \mathrm{EH}$ will be equal to $k$.

It may be noticed here that although the transformation was originally defined analytically, a purely geometrical form may be given to it; for (fig. 6) the conditions

$$
\tan \frac{1}{2} \mathrm{RPE}=k \tan \frac{1}{2} \mathrm{QPE} \text { and } \mathrm{CE}=k \mathrm{ED} \text { are identical. }
$$

Expression for the radius of the transformed circle.

In fig. 6 let $\mathrm{AE}=d, \mathrm{AH}=r, \mathrm{BE}=d^{\prime}, \mathrm{BK}=r^{\prime}$; then by the property of the radical axis

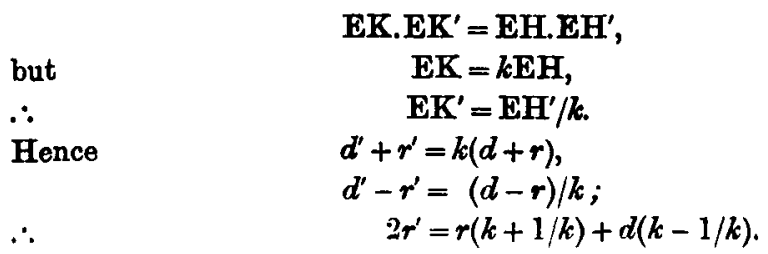


Hence the radius of the transformed circle will be zero, or this circle will reduce to a point, if

$$
k^{2}=(d-r) /(d+r)
$$

which gives real values for $k$ if the given circle does not cut the axis of transformation.

Another demonstration may be obtained by using a special kind of co-ordinates. Thus (fig. 7) consider a oircle of radius $a$, the distance of the centre of which from a fixed axis is $d$. Let the tangent at any point $P$ make an angle $\theta$ with the axis, and let $r$ be the length of the part of this tangent intercepted by the axis; then

$$
\begin{aligned}
& r \sin \theta=d+a \cos \theta, \\
& \therefore \quad r=d \operatorname{cosec} \theta+a \cot \theta,
\end{aligned}
$$

is the equation to the circle in this system of co-ordinates.

This equation may be written

$$
r=d\left(1+\tan ^{2} \frac{1}{2} \theta\right) / 2 \tan \frac{1}{2} \theta+a\left(1-\tan ^{2} \frac{1}{2} \theta\right) / 2 \tan \frac{1}{2} \theta
$$

or, $\quad r=\left\{(d+a)+(d-a) \tan ^{2} \frac{1}{2} \theta\right\} / 2 \tan \frac{1}{2} \theta$.

The equation is transformed by writing

$$
k \tan \frac{1}{2} \phi \text { in place of } \tan \frac{1}{2} \theta .
$$

$$
\text { This gives } \begin{aligned}
r & =\left\{(d+a)+(d-a) k^{2} \tan ^{2} \frac{1}{2} \phi\right\} / 2 k \tan \frac{1}{2} \phi \\
\text { or } \quad r & =\left\{(d+a) / k+(d-a) k \tan ^{2} \frac{1}{2} \phi\right\} / 2 \tan \frac{1}{2} \phi,
\end{aligned}
$$

which represents a circle. If $\delta$ be the distance of its centre from the axis and $a$ its radius, then

$$
\begin{aligned}
& \delta+u=(d+a) / k, \\
& \delta-a=(d-a) k,
\end{aligned}
$$

results which agree with those obtained by the former method.

The principal characteristic of this method of transformation is that the length of the tangent from a point in the axis remains unaltered. This is obvious in the case of the circle from the demonstration given above; and, in the case of any other curve, may be seen from the fact that, if a circle be drawn to touch the curve at its point of contact with a given tangent, and the whole figure be transformed, the transformed circle will touch the other transformed 
curve at its point of contact with the line into which the given tangent transforms. A direct proof of this property by means of infinitesimals may also be given, as follows:-

Let $\mathrm{AP}, \mathrm{BP}$, (fig. 8) be two consecutive tangents; $\mathrm{AQ}, \mathrm{BQ}$, the transformed tangents; let $\mathrm{PAX}=\theta, \mathrm{QAX}=\phi$; draw $\mathrm{AN}$ and $\mathrm{AM}$ perpendicular to $B P$ and $B Q$ respectively ; then

$$
\mathrm{AP}=\mathrm{AN} / \mathrm{APB}=\mathrm{AN} / d \theta=\mathrm{AB} \sin \theta / d \theta .
$$

Now, $\quad \tan \frac{1}{2} \phi=k \tan \frac{1}{2} \theta, \therefore \sec ^{2} \frac{1}{2} \phi d \phi=k \sec ^{2} \frac{1}{2} \theta d \theta$;

$$
\begin{aligned}
\therefore \mathrm{AP} & =\mathrm{AB} \sin \theta / d \theta \\
& =2 \mathrm{AB} \tan \frac{1}{2} \theta / \sec ^{2} \frac{1}{2} \theta d \theta \\
& =2 \mathrm{AB} \tan \frac{1}{2} \phi / \sec \frac{1}{2} \phi d \phi . \\
& =\mathrm{AQ} .
\end{aligned}
$$

If a tangent to a given curve $\mathrm{P}$ makes an angle $\theta$ with a given axis, it may also be considered as making an angle $\pi+\theta$ with that axis. Hence if the angle of inclination $\phi$ of the transformed tangent is determined by the relation $\tan \frac{1}{2} \phi=k \tan \frac{1}{2} \theta$, there are two possible values of $\phi$, and therefore two possible positions of the transformed tangent. Each of these tangents has an envelope, and hence a curve may, in general, be transformed in two different ways for the same value of $k$. In the case of the circle, these two transformations may easily be discussed geometrically; the following is an analytical investigation.

Let the co-ordinates of any point on the circle (figs. 9 and 10) be expressed in terms of the angle $\theta$ contained by a radius to the point and a radius parallel to the axis of transformation, namely

$$
x=a+r \cos \theta, y=b+r \sin \theta \text {. }
$$

Let $\phi$ be the angle made by a tangent to the circle with the axis of transformation, and let $\tan \frac{1}{2} \psi=k \tan \frac{1}{2} \phi$.

In fig. $9, \phi=\theta+\frac{\pi}{2}$; in fig. $10, \phi=\theta+\frac{3 \pi}{2}$. Consider fig. 9 .

$$
\begin{aligned}
& x=a+r \cos \theta=a+r \sin \phi \\
& y=b+r \sin \theta=b-r \cos \phi
\end{aligned}
$$

The equation to the tangent at $\left(x_{1}, y_{1}\right)$ is

$$
y-y_{1}=\left(x-x_{1}\right) \tan \phi \text {. }
$$


When $y=0$

$$
x^{\prime}=x_{1}-y_{1} \cot \phi
$$

and the transformed curve is the envelope of

that is

$$
y=\left(x-x^{\prime}\right) \tan \psi, \text { where } \tan \frac{1}{2} \psi=k \tan \frac{1}{2} \phi ;
$$

$$
\begin{aligned}
y & =\left(x-x_{1}+y_{1} \cot \phi\right) \tan \psi \\
& =(x-a+b \cot \phi-r \operatorname{cosec} \phi) \tan \psi .
\end{aligned}
$$

If $\tan \frac{1}{2} \phi=\lambda$, this equation reduces to

$$
\left\{k^{2} y-(r+b) k\right\} \lambda^{2}+2(x-a) k \lambda+(b-r) k-y=0 ;
$$

the envelope of which is given by

that is,

$$
(x-a)^{2} k^{2}-\left\{k^{2} y-(r+b) k\right\}\{(b-r) k-y\}=0
$$

$$
(x-a)^{2}+\left\{y-\frac{1}{2} b(k+1 / k)+\frac{1}{2} r(k-1 / k)\right\}^{2}=\frac{1}{4}\{b(k-1 / k)-r(k+1 / k)\}^{2} .
$$

To get the equation for the case of fig. 10, the sign of $r$ must be changed throughout.

Let $\delta$ and $\rho$ be the distance of the centre from the axis and the radius for fig. 9, and $\delta^{\prime}$ and $\rho^{\prime}$ the corresponding quantities for fig. 10. Then

$$
\begin{array}{cc}
\delta=\frac{1}{2} b(k+1 / k)-\frac{1}{2} r(k-1 / k) ; & \delta^{\prime}=\frac{1}{2} b(k+1 / k)+\frac{1}{2} r(k-1 / k) ; \\
\rho=\frac{1}{2} b(k-1 / k)-\frac{1}{2} r(k+1 / k) ; & \rho^{\prime}=\frac{1}{2} b(k-1 / k)+\frac{1}{2} r(k+1 / k) . \\
\delta+\rho=(b-r) k ; & \delta^{\prime}+\rho^{\prime}=(b+r) k ; \\
\delta-\rho=(b+r) / k ; & \delta^{\prime}-\rho^{\prime}=(b-r) / k .
\end{array}
$$

Hence, for any given value of $k$, a circle $A$ may be transformed into two distinct circles $B$ and $C$; the difference being that for one of the circles, $B$ say, the tangent which envelopes $A$ is supposed to move round $A$ in the positive direction, while for $C$ the enveloping tangent moves round $A$ in the negative direction. Only one of the circles $B$ and $\mathrm{O}$ may be considered at a time, or both at once, as is convenient. The two forms of transformation may be distinguished as direct and inverse.

In fig. 11, $\mathrm{A}$ and $\mathrm{B}$ are two circles whose direct common tangents intersect in the axis of transformation. A transforms into the two circles $\mathbf{O}$ and $O^{\prime}$, and $\mathrm{B}$ into $\mathrm{D}$ and $\mathrm{D}^{\prime}$. As is shown in the figure, a direct common tangent to $A$ and $B$ transforms into a direct common tangent to $C$ and $D$, and also into a direct common tangent to $\mathbf{C}^{\prime}$ and $D^{\prime}$; while an inverse common tangent to $A$ and $B$ transforms 
into a common tangent to $\mathrm{C}$ and $\mathrm{D}^{\prime}$ and also into a common tangent to $\mathrm{C}^{\prime}$ and $\mathrm{D}$; that is to say, if two circles are transformed either both directly or both inversely, the direct common tangents transform into common tangents, while if one of the circles is transformed directly and the other inversely, the inverse common tangents transform into common tangents. It is obvious that when two circles are transformed the length of the common tangent remains unaltered. This corresponds to the fact that, in transformation by similar figures, the angle of intersection of two curves remains unaltered.

It was shown that, hy selection of a proper value of $k$, any circle may be transformed into a point. In this case any two tangents from a point in the axis of transformation transform into the same straight line; and hence any two circles, the common tangents to which intersect in the axis of transformation may be transformed into points by the same transformation. If an axis of similitude is taken for axis of transformation, any three circles may be transformed into points ; and in general any number of circles which possess a common axis of similitude may be so transformed, two circles that lie on the same side of the axis being transformed either both directly or both inversely, and two that lie on opposite sides being transformed, one directly and one inversely. It may be noted that a point transforms into only one circle.

If a single circle is transformed in all possible ways, that is, for all values of $k$, an infinite series of circles is obtained, consisting of all the circles that cut another system orthogonally. If this system is transformed for any one value of $k$, it transforms into itself.

If all the points on the circumference of a circle be transformed, the centres of the circles into which they transform will lie on an ellipse.

Let $\mathbf{P}$ (fig. 12) transform into a circle of which the centre is $\mathbf{P}^{\prime}$, and which passes through $Q$ and $R$,

then

$$
\begin{array}{r}
\mathrm{QM}=\mathrm{PM} / k \text { and } \mathbf{R M}=k \mathbf{P M} \\
\therefore \mathrm{QM}+\mathbf{R M}=(k+1 / k) \mathbf{P M} \\
\therefore \mathrm{P}^{\prime} \mathrm{M}=\frac{1}{2}(k+1 / k) \mathbf{P M} .
\end{array}
$$

Hence the locus of $\mathbf{P}^{\prime}$ is an ellipse, one of the axes of which is equal to the diameter of the circle on which $P$ lies. The other axis is $\frac{1}{2}(k+1 / k)$ times this diameter. 
Exadiples of the Application of this Method.

(1) By means of this method of transformation may be found very easily the condition that four straight lines touch the same circle, in terms of the angles that the lines make with the third diagonal of the quadrilateral formed by them.

Let $\mathrm{AB}$ (fig. 13) be this third diagonal ; and let the circle touched by the four lines be transformed into the point $P$.

Let $\mathrm{PA}$ and $\mathrm{PB}$ make angles $\alpha$ and $\beta$ with $\mathrm{AB}$.

Let the two tangents through $A$ make angles $\phi_{1}$ and $\phi_{2}$, and the two tangents through $\mathrm{B}$ angles $\psi_{1}$ and $\psi_{2}$, with $\mathrm{AB}$.

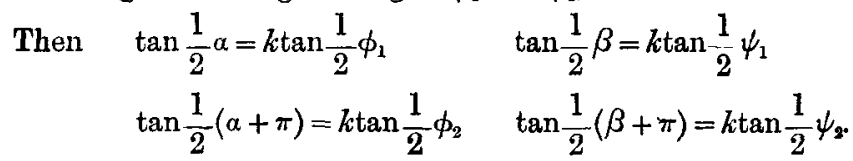

The required condition is obtained at once, by elimination of $\alpha, \beta$ and $k$ from these four equations; namely,

$$
{ }^{*} \tan \frac{1}{2} \phi_{1} \tan \frac{1}{2} \phi_{2}=\tan \frac{1}{2} \psi_{1} \tan \frac{1}{2} \psi_{2} \text {. }
$$

(2) If four circles have a common axis of similitude, they may be transformed into four points. Hence their six common tangents satisfy the relation which connects the six straight joining four points.

It should be noted here, and the remark applies to all cases in which an axis of similitude and a common tangent are referred to, that when the axis of similitude passes through the external centre of similitude of two circles, the direct common tangent is to be understood, and when through the internal centre of similitude the inverse common tangent.

(3) If four circles have a common axis of similitude, and touch a common circle, their common tangents satisfy the relation connecting the diagonals and sides of a quadrilateral inscribed in a circle, namely,

$$
h^{2}=(a c+b d)(a d+b c) /(a b+c d), k^{2}=(a c+b d)(a b+c d) /(a d+b c) .
$$

(4) Given three fixed circles A, B, C, the locus of the centre of a circle $P$ such that the common tangents to $P$ and $A, P$ and $B, P$

* This result is implicitly contained in a paper by me in vol. iii. of the Proceedings, page 60. 
and $\mathrm{C}$, are equal is a straight line perpendicular to the axis of similitude of the circles $\mathrm{A}, \mathrm{B}, \mathrm{C}$.

This result is obtained by transforming the circle $A, B, C$, into three points, and noticing that the system of circles $P$ transforms into a system of concentric circles.

There are four different cases, one arising from consideration of each of the axes of similitude.

The common tangent to either of the circles $\mathrm{A}, \mathrm{B}, \mathrm{C}$, and the circle $P$ is a maximum when $P, A, B, C$ have a common axis of similitude.

(5) The eight circles touching the circles A, B, C, of the last example are particular cases of the circle $\mathbf{P}$. Hence the centres of these eight circles lie in pairs on straight lines, passing through the radical centre and perpendicular to the axes of sinilitude of the circles A, B, C.

The two circles that have their centres in the same line perpendicular to one of the axes of similitude have obviously that axis for their radical axis. (See Casey's Sequel to Euclid, Third Edition, page 121.)

(6) The last example gives a method for constructing the circles touching three given circles.

(7) Let three points be transformed with every possible value of $k$. A one-fold infinity of systems of three circles will be obtained, possessing the properties that one of the axes of similitude of all the systems and the centres of similitude lying in that axis are fixed, and that the lengths of the tangents to the circles from the fixed centres of similitude are invariable. The radical centre and the centres of the common tangent circles for all the systems will lie in a straight line perpendicular to the fixed axis of similitude. [To each axis of similitude correspond two tangent circles.]

(8) The locus of the centres of all the circles that touch a given circle and have a common axis of similitude is an ellipse. 